Pacific Journal of 


\section{THE PTOLEMAIC INEQUALITY IN HILBERT GEOMETRIES}

\section{DAVID C. KAY}

Let $M$ be a metric space, and if $x$ and $y$ are points in $M$, let $x y$ denote the metric. The space $M$ and its metric are called ptolemaic if for each quadruple of points $x_{i}(i=1,2,3,4)$ the ptolemaic inequality

$$
x_{1} x_{2} \cdot x_{3} x_{4}+x_{1} x_{3} \cdot x_{2} x_{4} \geqq x_{1} x_{4} \cdot x_{2} x_{3}
$$

holds. If the inequality holds only in some neighborhood of each point the space and its metric are said to be locally ptolemaic. Euclidean space is known to be ptolemaic and therefore, locally ptolemaic. We are interested here in certain non-euclidean spaces which may possibly be locally ptolemaic. The author has proved in his thesis Michigan State University Doctoral Dissertation, 1963) that a Riemannian geometry is locally ptolemaic if and only if it has nonpositive curvature, and that a Finsler space which is locally ptolemaic is Riemannian. The main result established here extends the theorem regarding Finsler spaces to include Hilbert geometries as well: A Hilbert geometry is locally ptolemaic if and only if it is hyperbolic.

The ptolemaic inequality is related to problems of curvature in metric geometry. Assuming this condition enables one to prove that a curve is a geodesic if and only if its metric curvature is zero at each of its points (see [3]). Blumenthal has investigated a number of properties peculiar to ptolemaic metric spaces in [2]. It is then significant to determine what metric spaces are ptolemaic. A question which remains unsettled is whether a non-Riemannian $G$-space (Busemann [4, p. 37]) can be locally ptolemaic. The result obtained here concerning Hilbert geometries, together with several in the author's thesis lends support to the conjecture that such a space does not exist.

Hilbert geometry is a generalization of the well-known Klein model for hyperbolic geometry. Consider an arbitrary bounded convex body $C$ with nonempty interior $D$ in euclidean space. If $x$ and $y$ be any two points in $D$, a distance function may be defined as

$$
h(x, y)=k|\log R(x y, a b)|
$$

where $k$ is a positive constant, $a$ and $b$ are the points of intersection of $C$ with the affine line $L_{x y}$ determined by $x$ and $y$, and $R(x y, a b)$ is 
the usual (euclidean) cross-ratio. It can be proved that $h(x, y)$ is a metric, and moreover if $C$ is either strictly convex or has the property that all linear triples which it contains belong to the same hyperplane, linearity under $h$ coincides with linearity under the euclidean metric. Hence, the geodesics for $h$ are the portions of affine lines contained in $D$. The function $h$ is then called a Hilbert metric for $D$ and with this metric, $D$ is called a Hilbert geometry.

Let $p$ be any point in $D$. With the euclidean metric understood, let $C^{\prime}$ be the reflection of $C$ in $p$. Each ray $r$ from $p$ will cut $C$ and $C^{\prime}$ in unique points $x_{r}$ and $x_{r}^{\prime}$ respectively. Define $U_{p}$ as the set of all points $u_{r}$ such that $u_{r} \in r^{\prime}$ and

$$
\frac{2}{\overline{p u}_{r}}=\frac{1}{\overline{p x}_{r}}+\frac{1}{\overline{p x}_{r}^{\prime}},
$$

where $\overline{x y}$ denotes the euclidean metric. It is clear that $U_{p}$ is symmetric about $p$. With our former assumptions on $C$ we can convince ourselves that $U_{p}$ is strictly convex. Suppose rays $r, s$, and $t$ with origin $p$ meet $C$ in $x, y$, and $z$, and $C^{\prime}$ in $x^{\prime}, y^{\prime}$, and $z^{\prime}$. If further, $u, v$, and $w$ are the intersections of those rays with $U_{p}$ we may use (1) to deduce

$$
R\left(p u, x x^{\prime}\right)=R\left(p v, y y^{\prime}\right)=R\left(p w, z z^{\prime}\right)=-1 .
$$

Assume that $v_{1}$ is a point on the segment joining $u$ and $w, S_{u w}$, and that $s$ passes through $v_{1}$. Finally, let $y_{1}=s \times S_{x z}$ and $y_{1}^{\prime}=s \times S_{x^{\prime} z^{\prime}}$. Now, $q=L_{x z} \times L_{x^{\prime} z^{\prime}}$ is the center of a projectivity which maps $x$ into $z, u$ into $w$, and $x^{\prime}$ into $z^{\prime}$. It also maps $x, u$, and $x^{\prime}$ into $y_{1}, v_{1}$, and $y_{1}^{\prime}$, so

$$
R\left(p v_{1}, y_{1} y_{1}^{\prime}\right)=-1
$$

which is equivalent to

$$
\frac{2}{\overline{p v}_{1}}=\frac{1}{\overline{p y}_{1}}+\frac{1}{\overline{p y}_{1}^{\prime}}
$$

Since $C$ and $C^{\prime}$ are convex we have $\overline{p y}_{1} \leqq \overline{p y}$ and $\overline{p y}_{1}^{\prime} \leqq \overline{p y}^{\prime}$, with strict inequality in at least one case. Hence

$$
1 / \overline{p y}_{1}+1 / \overline{p y}_{1}^{\prime}>1 / \overline{p y}+1 / \overline{p y}^{\prime}=2 / \overline{p v} .
$$

Therefore, $\overline{p v}_{1}<\overline{p v}$ proving that $U_{p}$ is strictly convex.

As proved in [5], it follows that $U_{p}$ is the unit sphere of a Minkowski metric $m_{p}(x, y)$ defined on $D$ having the same geodesics as h. Further, 


$$
\lim _{x, y \rightarrow p} \frac{h(x, y)}{m_{p}(x, y)}=1 .
$$

In this sense a Hilbert metric is "locally" Minkowskian. The metric $m_{p}(x, y)$ is called the associated local Minkowski metric of $h(x, y)$ at $p$.

1. Perpendicularity in Hilbert geometries. Suppose $D$ is metrized as a straight space by the metric $d(x, y)$. We shall say that a geodesic $L_{1}$ is perpendicular to another geodesic $L_{2}$ at $p \in L_{1} \cap L_{2}$ whenever $x \in L_{1}$ and $y \in L_{2}$ implies

$$
d(x, p) \leqq d(x, y) .
$$

This condition will be denoted $L_{1} \perp_{d} L_{2}$. The problem of existence has been solved in [4, pp. 119-122]: Given a geodesic $L_{2}$ and a point $q$ not on it there exists a unique geodesic $L_{1}$ such that $q \in L_{1}$ and $L_{1} \perp_{d} L_{2}$ at some point $p$, and if $p$ is any point on $L_{2}$ and $H$ is any planar section of $D$ containing $L_{2}(H$ is then a two-dimensional straight space) then precisely one geodesic $L_{1}$ exists in $H$ such that $L_{1} \perp_{d} L_{2}$ at $p$. Since the Hilbert metric and the metrics $m_{p}(x, y)$ for $p \in D$ metrize $D$ as a straight space this statement applies to those metrics.

Perpendicularity is called symmetric if for any two geodesics $L_{1}$ and $L_{2}, L_{1} \perp_{d} L_{2}$ implies $L_{2} \perp_{d} L_{1}$. The following theorem was proved in [6]:

THEOREM 1. Perpendicularity is symmetric in a Hilbert geometry if and only if it is hyperbolic.

Of significance to us is

THeOREM 2. At any point in $D$, perpendicularity under the local Minkowski metric coincides with perpendicularity under the original Hilbert metric.

Proof. Let $L_{1} \perp_{h} L_{2}$ at $p \in D$ and suppose $x \in L_{1}, y \in L_{2}, x \neq p$, and $y \neq p$. With $p$ as origin, define the points $x_{\lambda}=\lambda x$ and $y_{\lambda}=\lambda y$ for each real $\lambda, 0<\lambda<1$, where $\lambda x$ denotes the usual scalar multiplication in euclidean space. Under the local Minkowski metric $m(x, y)$ at $p$, the triangles determined by the triples $(p, x, y)$ and $\left(p, x_{\lambda}, y_{\lambda}\right)$ are similar and thus $m(x, p) / m\left(x_{\lambda}, p\right)=m(x, y) / m\left(x_{\lambda}, y_{\lambda}\right)$. Since $h\left(x_{\lambda}, p\right) \leqq h\left(x_{\lambda}, y_{\lambda}\right)$ for each $\lambda$ we have

$$
\frac{h\left(x_{\lambda}, p\right)}{m\left(x_{\lambda}, p\right)} \cdot m(x, p) \leqq \frac{h\left(x_{\lambda}, y_{\lambda}\right)}{m\left(x_{\lambda}, y_{\lambda}\right)} \cdot m(x, y) .
$$

But $\lim _{\lambda \rightarrow 0}\left[h\left(x_{\lambda}, p\right) / m\left(x_{\lambda}, p\right)\right]=\lim _{\lambda \rightarrow 0}\left[h\left(x_{\lambda}, y_{\lambda}\right) / m\left(x_{\lambda}, y_{\lambda}\right)\right]=1$ so it fol- 
lows that $m(x, p) \leqq m(x, y)$ and therefore $L_{1} \perp_{m} L_{2}$ at $p$.

Conversely, suppose $L_{1} \perp_{m} L_{2}$ at $p$. Consider $L_{1}^{\prime}$ the geodesic which passes through $p$, lies in the plane determined by $L_{1}$ and $L_{2}$, such that $L_{1}^{\prime} \perp_{h} L_{2}$ at $p$. Then, from the preceding case, $L_{1}^{\prime} \perp_{m} L_{2}$ at $p$. Since the geodesic perpendicular to $L_{2}$ at $p$ is unique in this plane, $L_{1}^{\prime}=L_{1}$. Therefore $L_{1} \perp_{h} L_{2}$ at $p$.

If a Hilbert geometry has the property that the local Minkowski metric at each point is euclidean (that is, its unit sphere is an ellipsoid) we shall say it is locally euclidean. We have an immediate corollary, making use of Theorem 1:

Corollary 1. A locally euclidean Hilbert geometry is hyperbolic.

2. Ptolemaic metrics. In [9] Schoenberg has proved that a ptolemaic normed linear space is an inner product space. We may state this in the more pertinent form:

THEOREM 3. A Minkowski metric is euclidean if and only if it is ptolemaic.

This enables us to prove

THEOREM 4. A locally ptolemaic Hilbert geometry is hyperbolic.

Proof. It will be shown that the given Hilbert metric $h(x, y)=$ $x y$ is locally euclidean. The rest follows immediately from Corollary 1. Let $p$ be any point in $D$ and suppose $m(x, y)=\overline{x y}$ is the associated local Minkowski metric at $p$. In view of Theorem 3 it suffices to show that $\overline{x y}$ is ptolemaic. Let $x, y, z$, and $w$ be four points in $D$ and let $N$ be the neighborhood about $p$ in which the Hilbert metric is ptolemaic. As before, with $p$ as origin define $x_{\lambda}=\lambda x, y_{\lambda}=\lambda y$, $z_{\lambda}=\lambda z, w_{\lambda}=\lambda w$ for $\lambda$ a positive real number. For all sufficiently small $\lambda$ the points $x_{\lambda}, y_{\lambda}, z_{\lambda}$, and $w_{\lambda}$ lie in $N$ and we therefore have

$$
x_{\lambda} y_{\lambda} \cdot z_{\lambda} w_{\lambda}+x_{\lambda} z_{\lambda} \cdot y_{\lambda} w_{\lambda} \geqq x_{\lambda} w_{\lambda} \cdot y_{\lambda} z_{\lambda}
$$

or,

$$
\begin{aligned}
& \frac{x_{\lambda} y_{\lambda} \cdot z_{\lambda} w_{\lambda}}{\overline{x_{\lambda} y_{\lambda}} \cdot \overline{z_{\lambda} w_{\lambda}}} \cdot \frac{\overline{x_{\lambda} w_{\lambda}} \cdot \overline{y_{\lambda} z_{\lambda}}}{x_{\lambda} w_{\lambda} \cdot y_{\lambda} z_{\lambda}} \cdot \frac{\overline{x_{\lambda} y_{\lambda}} \cdot \overline{z_{\lambda} w_{\lambda}}}{\overline{x_{\lambda} w_{\lambda}} \cdot \overline{y_{\lambda} z_{\lambda}}} \\
+ & \frac{x_{\lambda} z_{\lambda} \cdot y_{\lambda} w_{\lambda}}{\overline{y_{\lambda} z_{\lambda}} \cdot \overline{y_{\lambda} w_{\lambda}}} \cdot \frac{\overline{x_{\lambda} w_{\lambda}} \cdot \overline{y_{\lambda} z_{\lambda}}}{x_{\lambda} w_{\lambda} \cdot y_{\lambda} z_{\lambda}} \cdot \frac{\overline{x_{\lambda} z_{\lambda}} \cdot \overline{y_{\lambda} w_{\lambda}}}{\overline{x_{\lambda} w_{\lambda}} \cdot \overline{y_{\lambda} z_{\lambda}}} \geqq 1 .
\end{aligned}
$$

Since $u \rightarrow \lambda u=u_{\lambda}$ is a similarity mapping we have 


$$
\begin{aligned}
& \frac{x_{\lambda} y_{\lambda} \cdot z_{\lambda} w_{\lambda}}{\overline{x_{\lambda} y_{\lambda}} \cdot \overline{z_{\lambda} w_{\lambda}}} \cdot \frac{\overline{x_{\lambda} w_{\lambda}} \cdot \overline{y_{\lambda} z_{\lambda}}}{x_{\lambda} w_{\lambda} \cdot y_{\lambda} z_{\lambda}} \cdot \frac{\overline{x y} \cdot \overline{z w}}{\overline{x w} \cdot \overline{y z}} \\
+ & \frac{x_{\lambda} z_{\lambda} \cdot y_{\lambda} w_{\lambda}}{\overline{x_{\lambda} z_{\lambda}} \cdot \overline{y_{\lambda} w_{\lambda}}} \cdot \frac{\overline{x_{\lambda} w_{\lambda}} \cdot \overline{y_{\lambda} z_{\lambda}}}{x_{\lambda} w_{\lambda} \cdot y_{\lambda} z_{\lambda}} \cdot \frac{\overline{x z} \cdot \overline{y w}}{\overline{x w} \cdot \overline{y z}} \geqq 1 .
\end{aligned}
$$

Taking the limit in (3) as $\lambda \rightarrow 0$ yields

$$
\frac{\overline{x y} \cdot \overline{z w}}{\overline{x w} \cdot \overline{y z}}+\frac{\overline{x z} \cdot \overline{y w}}{\overline{x w} \cdot \overline{y z}} \geqq 1,
$$

the desired inequality.

If $x, y$, and $z$ are any three points in $D$ and $m$ is the midpoint of segment $S_{y z}$, the euclidean formula for the median $S_{x m}$ is

$$
(x m)^{2}=(x y)^{2} / 2+(x z)^{2} / 2-(y z)^{2} / 4 .
$$

It can be shown that for a hyperbolic metric, instead of (4), we have

$$
(x m)^{2} \leqq(x y)^{2} / 2+(x z)^{2} / 2-(y z)^{2} / 4
$$

with equality only when $x, y$, and $z$ are linear. Inequality (5) in turn implies the ptolemaic inequality as we shall see. This fact may then be used to derive the ptolemaic inequality in hyperbolic geometry.

We shall use (5) to derive the more general inequality

$$
(x m)^{2} \leqq \lambda(x y)^{2}+(1-\lambda)(x z)^{2}-\lambda(1-\lambda)(y z)^{2}, \quad 0 \leqq \lambda \leqq 1,
$$

for any point $m$ on $S_{y z}$ with $\lambda=m z / y z$. Induction will establish $\left(5^{\prime}\right)$ for $\lambda$ a diadic rational $\mu / 2^{\nu}$ where $\mu$ and $\nu$ are nonnegative integers and $0 \leqq \mu \leqq 2^{\nu}$. The inequality is clear for the case $\nu=1$. Assume it has been proved for all diadic rationals of the form $\mu / 2^{\text {k }}$, $\kappa<\nu$, and let $\mu / 2^{\nu}$ be given, excluding the cases $\mu=0, \mu=2^{\nu}$, and $\mu$ even, as trivial. Then, there exist points $y^{\prime}$ and $z^{\prime}$ on $S_{y z}$ such that $(\mu+1) / 2^{\nu}=y^{\prime} z / y z$ and $(\mu-1) / 2^{\nu}=z^{\prime} z / y z$. Since $\mu$ is odd, $(\mu+1) / 2^{\nu}$ and $(\mu-1) / 2^{\nu}$ are diadic rationals of the form $\mu^{\prime} / 2^{\kappa}$ for $\kappa<\nu$ and the induction hypothesis implies

$$
\begin{aligned}
\left(x y^{\prime}\right)^{2} \leqq & \frac{\mu^{2}+1}{2^{\nu}}(x y)^{2} \\
& +\left(1-\frac{\mu+1}{2^{\nu}}\right)(x z)^{2}-\frac{\mu+1}{2^{\nu}}\left(1-\frac{\mu+1}{2^{\nu}}\right)(y z)^{2} \\
\left(x z^{\prime}\right)^{2} \leqq & \frac{\mu-1}{2^{\nu}}(x y)^{2} \\
& +\left(1-\frac{\mu-1}{2^{\nu}}\right)(x z)^{2}-\frac{\mu-1}{2^{\nu}}\left(1-\frac{\mu-1}{2^{\nu}}\right)(y z)^{2} .
\end{aligned}
$$

Since $\mu / 2^{\nu}=m z / y z, m$ is the midpoint of the segment $S_{y^{\prime} z^{\prime}}$ and (5) 
yields

$$
(x m)^{2} \leqq\left(x y^{\prime}\right)^{2} / 2+\left(x z^{\prime}\right)^{2} / 2-\left(y^{\prime} z^{\prime}\right)^{2} / 4
$$

Substituting $y z / 2^{\nu-1}$ for $y^{\prime} z^{\prime}$ and making use of (6), (7) becomes

$$
(x m)^{2} \leqq \frac{\mu}{2^{\nu}}(x y)^{2}+\left(1-\frac{\mu}{2^{\nu}}\right)(x z)^{2}-\frac{\mu}{2^{\nu}}\left(1-\frac{\mu}{2^{\nu}}\right)(y z)^{2}
$$

and induction carries. Then $\left(5^{\prime}\right)$ is true for arbitrary real $\lambda, 0 \leqq \lambda \leqq 1$.

THEOREM 5. The ptolemaic inequality holds in any metric space $D$ in which the inequality (5) holds, provided the metric is complete and convex.

Proof. The completeness and convexity of the metric guarantees the existence of segments. Moreover, (5) implies the uniqueness of the segment joining each pair of points. Let $x, y, z$, and $w$ be any four points in $D$. We must prove that

$$
x y \cdot z w+x z \cdot y w \geqq x w \cdot y z .
$$

If the four points are not distinct, (8) follows immediately; hence we set aside this case, as well as the case when the four points lie on a segment. In a euclidean plane, the euclidean metric denoted $\overline{p q}$, let $x^{\prime}, y^{\prime}$, and $z^{\prime}$ be the vertices of a triangle such that $\overline{x^{\prime} y^{\prime}}=x y, \overline{x^{\prime} z^{\prime}}=$ $x z$, and $\overline{y^{\prime} z^{\prime}}=y z$, and let $w^{\prime}$ be a point such that $\overline{y^{\prime} w^{\prime}}=y w, \overline{w^{\prime} z^{\prime}}=$ $w z$, and the segment $S_{x^{\prime} w^{\prime}}$ has at least one point $m^{\prime}$ in common with the line $L_{y^{\prime} z^{\prime}}$. Point $m^{\prime}$ is determined uniquely since the linearity of $x^{\prime}, y^{\prime}, z^{\prime}$, and $w^{\prime}$ would imply that $x, y, z$, and $w$ lie on a metric segment (see [4] p. 29).

Case 1. $m^{\prime}$ lies on $S_{y^{\prime} z^{\prime}}$. Locate $m$ in $D$ such that $m$ lies on the metric segment joining $y$ and $z$, and $m z=\overline{m^{\prime} z^{\prime}}$. Put $\lambda=m z / y z=$ $\overline{m^{\prime} z^{\prime}} \overline{y^{\prime} z^{\prime}}$. Applying $\left(5^{\prime}\right)$,

$$
\begin{aligned}
(x m)^{2} & \leqq \lambda(x y)^{2}+(1-\lambda)(x z)^{2}-\lambda(1-\lambda)(y z)^{2} \\
& =\lambda\left(\overline{x^{\prime} y^{\prime}}\right)^{2}+(1-\lambda)\left(\overline{x^{\prime} z^{\prime}}\right)^{2}-\lambda(1-\lambda)\left(\overline{y^{\prime} z^{\prime}}\right)^{2} \\
& =\left(\overline{m^{\prime} x^{\prime}}\right)^{2}
\end{aligned}
$$

Therefore $x m \leqq \overline{m^{\prime} x^{\prime}}$ and similarly, $m w \leqq \overline{m^{\prime} w^{\prime}}$. Thus,

$$
x w \leqq x m+m w \leqq \overline{x^{\prime} m^{\prime}}+\overline{m^{\prime} w^{\prime}}=\overline{x^{\prime} w^{\prime}} .
$$

Apply the ptolemaic inequality for the euclidean metric

$$
\overline{x^{\prime} y^{\prime}} \cdot \overline{z^{\prime} w^{\prime}}+\overline{x^{\prime} z^{\prime}} \cdot \overline{y^{\prime} w^{\prime}} \geqq \overline{x^{\prime} w^{\prime}} \cdot \overline{y^{\prime} z^{\prime}}
$$


and we have

$$
x y \cdot z w+x z \cdot y w \geqq \overline{x^{\prime} w^{\prime}} \cdot y z \geqq x w \cdot y z .
$$

Case 2. $m^{\prime}$ is exterior to $S_{y^{\prime} z^{\prime}}$. We may assume that $\overline{x^{\prime} w^{\prime}}<x w$, for otherwise we could derive (8) just as we did above. Since $x^{\prime}, y^{\prime}, z^{\prime}$, and $w^{\prime}$ are not linear, at most one of the points $x^{\prime}, w^{\prime}$ lies on $L_{y^{\prime} z^{\prime}}$. We may suppose that $x^{\prime}$ does not lie on $L_{y^{\prime} z^{\prime}}$, for a reversing of the roles of $x$ and $w$ leaves (8) unchanged. Similarly, it may be assumed that $y^{\prime}$ is between $m^{\prime}$ and $z^{\prime}$. Let $K$ be the semicircle with center at $y^{\prime}$ and radius $\overline{x^{\prime} y^{\prime}}$ which passes through $x^{\prime}$ and whose base lies on $L_{y^{\prime} z^{\prime}}$. Further, let $u^{\prime}$ be the endpoint of $K$ lying on the same side of $y^{\prime}$ as $z^{\prime}$, and $v^{\prime}$ the point where the ray from $w^{\prime}$ through $y^{\prime}$ meets $K$. Since the sum of $\angle x^{\prime} y^{\prime} u^{\prime}$ and $\angle w^{\prime} y^{\prime} x^{\prime}$ is greater than or equal to $\pi, v^{\prime}$ lies on, or in the interior of, $\angle x^{\prime} y^{\prime} u^{\prime}$ and therefore belongs to $K^{\prime}$, the sub-arc of $K$ whose endpoints are $x^{\prime}$ and $u^{\prime}$. Now, every point $t^{\prime}$ on $K^{\prime}$ has the property that $\overline{t^{\prime} z^{\prime}} \leqq \overline{x^{\prime} z^{\prime}}$. Consider the continuous function $f\left(t^{\prime}\right)=$ $\overline{t^{\prime} w^{\prime}}, \quad t^{\prime} \in K^{\prime} . \quad f\left(x^{\prime}\right)=\overline{x^{\prime} w^{\prime}}<x w \quad$ and $\quad f\left(v^{\prime}\right)=\overline{v^{\prime} w^{\prime}}=\overline{w^{\prime} y^{\prime}}+\overline{y^{\prime} v^{\prime}}=$ $w y+y x \geqq x w$, so there exists a point $t^{\prime}$ on $K^{\prime}$ such that $\bar{t}^{\prime} \bar{w}^{\prime}=x w$. Applying the ptolemaic inequality to $t^{\prime}, y^{\prime}, z^{\prime}$, and $w^{\prime}$,

$$
\overline{t^{\prime} y^{\prime}} \cdot \overline{z^{\prime} w^{\prime}}+\overline{t^{\prime} z^{\prime}} \cdot \overline{y^{\prime} w^{\prime}}>\overline{t^{\prime} w^{\prime}} \cdot \overline{y^{\prime} z^{\prime}},
$$

which gives us

$$
\begin{aligned}
x y \cdot z w+x z \cdot y w \geqq x y \cdot z w+\overline{t^{\prime} z^{\prime}} \cdot y w \\
\quad=\overline{t^{\prime} y^{\prime}} \cdot \overline{z^{\prime} w^{\prime}}+\overline{t^{\prime} z^{\prime} \cdot \overline{y^{\prime} w^{\prime}}} \geqq \overline{t^{\prime} w^{\prime} \cdot \overline{y^{\prime} z^{\prime}}}=x w \cdot y z .
\end{aligned}
$$

Remark. This proof applies to $G$-spaces. Unfortunately, even in $G$-spaces, it is not known whether (5) characterizes the ptolemaic inequality. It is interesting to note in this connection that Blumenthal has investigated a property he calls the euclidean four-point property which is merely our $\left(5^{\prime}\right)$ (or its equivalent (5) in complete convex metric spaces) with equality prevailing (see [1]).

Theorem 5 then provides an easy proof of

THeorem 6. Hyperbolic geometry is ptolemaic.

Proof. If $x y$ is a hyperbolic metric, we observe from the cosine inequality (see [4, p. 268]) that if $x, y$, and $z$ are the vertices of a triangle and $m$ is the midpoint of $S_{y z}$, with $A$ one of the angles at $m$, then

$$
\begin{aligned}
& (x y)^{2} \geqq(x m)^{2}+(m y)^{2}-2(x m)(m y) \cos A \\
& (x z)^{2} \geqq(x m)^{2}+(m z)^{2}+2(x m)(m z) \cos A,
\end{aligned}
$$


and, since $m y=m z=y z / 2$, we have

$$
(x y)^{2}+(x z)^{2} \geqq 2(x m)^{2}+(y z)^{2} / 2,
$$

which is (5). By Theorem 5 the metric is ptolemaic.

Theorems 4 and 6 combine to give

Corollary 2. A Hilbert geometry is locally ptolemaic if and only if it is hyperbolic.

3. Related inequalities. In a $G$-space the ptolemaic inequality appears to be related to the "curvature" of the space. It can be easily verified that spherical geometry (a space having positive curvature) is not ptolemaic. This illustrates the theorem in the author's thesis that a Riemannian space is locally ptolemaic if and only if its curvature is nonpositive, which leads to the definition: A metric space has nonpositive curvature if and only if the ptolemaic inequality (8) holds locally. Other concepts of space curvature have been proposed. In [4, p. 237] Busemann defines nonpositive curvature as follows: If for each point there exists a neighborhood such that given any triple of points $(x, y, z)$ in that neighborhood, with $m_{y}$ the midpoint between $x$ and $y$, and $m_{z}$ the midpoint between $x$ and $z$, the inequality

$$
2 m_{y} m_{z} \leqq y z
$$

holds, the space is said to have nonpositive curvature. In his thesis the author proposes this definition (making use of inequality (5) above): If for each point there exists a neighborhood such that if $(x, y, z)$ be any triple of points in that neighborhood with $m$ the midpoint between $y$ and $z$ then

$$
(m x)^{2} \leqq(x y)^{2} / 2+(x z)^{2} / 2-(y z)^{2} / 4
$$

holds, the space is said to have nonpositive curvature. F. P. Pederson [8] has investigated still a different concept of nonpositive curvature and relates it to $(9)$.

Relatively little is known concerning the various implications which may exist among these concepts of curvature. The seemingly stronger (10) is shown to imply (9) in [4, pp. 268-269]. Our Theorem 5 shows that (10) implies (8) (locally). In Riemannian spaces they are each equivalent to nonpositive Riemannian curvature, but the situation is completely unsettled in Finsler geometry. In view of our Corollary 2 and the theorem of P. Kelly and E. Strauss [7] that a 
Hilbert geometry has nonpositive curvature in the sense of (9) if and only if it is hyperbolic, we may conclude (trivially) that the conditions (8), (9), and (10) are equivalent in Hilbert geometries. It would be of interest to determine further implications-if such existamong (8), (9), and (10) outside of Hilbert and Riemannian geometry.

\section{REFERENCES}

1. L. M. Blumenthal, Remarks concerning the Euclidean four-point property, Ergebnisse eines mathematischen Kolloquiums (Wien) 7 (1936), 7-10.

2. - Remarks on a Weak Four-Point Property, Revista de Ciencias, 45 (1943), $183-193$.

3. - Theory and Applications of Distance Geometry, Oxford University Press, London 1953.

4. H. Busemann, The Geometry of Geodesics, Academic Press, New York, 1957.

5. H. Busemann, and P. J. Kelly, Projective Geometry and Projective Metrics, Academic Press, New York, 1953.

6. P. J. Kelly, and L. J. Paige, Symmetric perpendicularity in Hilbert geometries, Pacific J. Math. 2 (1952), 319-322.

7. P. J. Kelly, and E. Straus, Curvature in Hilbert geometries, Pacific. J. Math. 8 (1958), 119-125.

8. F. P. Pederson, On spaces with negative curvature, Mat. Tidsskrift B, (1952), 6689.

9. I. J. Schoenberg, A Remark on M. M. Day's Characterization of Inner-Product Spaces and a Conjecture of L. M. Blumenthal, Proc. Amer. Math. Soc. 3 (1952), 961964.

Received April 27, 1965. Supported by a National Science Foundation Grant while the author was a Research Participant at the University of Oklahoma, summer 1964.

The UNIVERSity OF OKLAHOMA 



\section{PACIFIC JOURNAL OF MATHEMATICS}

\section{EDITORS}

H. SAMELSON

Stanford University

Stanford, California

J. P. JANS

University of Washington

Seattle, Washington 98105

\section{J. DugunduI}

University of Southern California Los Angeles, California 90007

RICHARD ARENS

University of California

Los Angeles, California 90024

\section{ASSOCIATE EDITORS}
E. F. BECKENBACH
B. H. NEUMANN
F. WOLF
K. YoSIDA

\section{SUPPORTING INSTITUTIONS}

\author{
UNIVERSITY OF BRITISH COLUMRIA \\ CALIFORNIA INSTITUTE OF TECHNOLOGY \\ UNIVERSITY OF CALIFORNIA \\ MONTANA STATE UNIVERSITY \\ UNIVERSITY OF NEVADA \\ NEW MEXICO STATE UNIVERSITY \\ OREGON STATE UNIVERSITY \\ UNIVERSITY OF OREGON \\ OSAKA UNIVERSITY \\ UNIVERSITY OF SOUTHERN CALIFORNIA
}

\author{
STANFORD UNIVERSITY \\ UNIVERSITY OF TOKYO \\ UNIVERSITY OF UTAH \\ WASHINGTON STATE UNIVERSITY \\ UNIVERSITY OF WASHINGTON \\ AMERICAN MATHEMATICAL SOCIETY \\ CHEVRON RESEARCH CORPORATION \\ TRW SYSTEMS \\ NAVAL ORDNANCE TEST STATION
}

Mathematical papers intended for publication in the Pacific Journal of Mathematics should be typewritten (double spaced). The first paragraph or two must be capable of being used separately as a synopsis of the entire paper. It should not contain references to the bibliography. Manuscripts may be sent to any one of the four editors. All other communications to the editors should be addressed to the managing editor, Richard Arens at the University of California, Los Angeles, California 90024 .

50 reprints per author of each article are furnished free of charge; additional copies may be obtained at cost in multiples of 50 .

The Pacific Journal of Mathematics is published monthly. Effective with Volume 16 the price per volume (3 numbers) is $\$ 8.00$; single issues, $\$ 3.00$. Special price for current issues to individual faculty members of supporting institutions and to individual members of the American Mathematical Society: $\$ 4.00$ per volume; single issues $\$ 1.50$. Back numbers are available.

Subscriptions, orders for back numbers, and changes of address should be sent to Pacific Journal of Mathematics, 103 Highland Boulevard, Berkeley 8, California.

Printed at Kokusai Bunken Insatsusha (International Academic Printing Co., Ltd.), No. 6, 2-chome, Fujimi-cho, Chiyoda-ku, Tokyo, Japan.

\section{PUBLISHED BY PACIFIC JOURNAL OF MATHEMATICS, A NON-PROFIT CORPORATION}

The Supporting Institutions listed above contribute to the cost of publication of this Journal, but they are not owners or publishers and have no responsibility for its content or policies. 


\section{Pacific Journal of Mathematics}

\section{Vol. 21, No. 2 December, 1967}

Arne P. Baartz, The measure algebra of a locally compact semigroup ..... 199

Robert F. Brown, On maps with identical fixed point sets............. 215

C. Buttin, Existence of a homotopy operator for Spencer's sequence in the analytic case ..................................... 219

Henry Werner Davis, An elementary proof that Haar measurable almost periodic functions are continuous ........................ 241

Zeev Ditzian, On asymptotic estimates for kernels of convolution transforms ...................................... 249

Robert E. Edwards, Boundedness principles and Fourier theory ......... 255

John A. Hildebrant, On compact unithetic semigroups ............... 265

Marinus A. Kaashoek and David Clark Lay, On operators whose Fredholm set is the complex plane ............................ 275

Sadao Kató, Canonical domains in several complex variables ........... 279

David Clifford Kay, The ptolemaic inequality in Hilbert geometries.... . . . 293

Joseph D. E. Konhauser, Biorthogonal polynomials suggested by the Laguerre polynomials ............................. 303

Kevin Mor McCrimmon, Macdonald's theorem with inverses .......... 315

Harry Eldon Pickett, Homomorphisms and subalgebras of multialgebras .................................... 327

Richard Dennis Sinkhorn and Paul Joseph Knopp, Concerning nonnegative matrices and doubly stochastic matrices ..............

Erling Stormer, On anti-automorphisms of von Neumann algebras ...

Miyuki Yamada, Regular semi-groups whose idempotents satisfy permutation identities .......................... 